\title{
Design and synthesis of novel spin-labeled camptothecin derivatives as potent cytotoxic agents
}

\author{
Xiao-Bo Zhao ${ }^{\mathrm{a}, \dagger}$, Dan Wu ${ }^{\mathrm{a}, \dagger}$, Mei-Juan Wang ${ }^{\mathrm{a}}$, Masuo Goto ${ }^{\mathrm{b}}$, Susan L. Morris-Natschke ${ }^{\mathrm{b}}$,

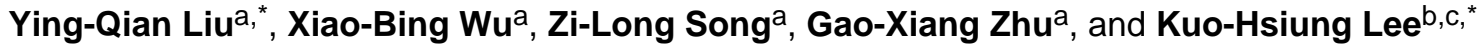 \\ aSchool of Pharmacy, Lanzhou University, Lanzhou 730000, PR China \\ bNatural Products Research Laboratories, UNC Eshelman School of Pharmacy, University of \\ North Carolina, Chapel Hill, North Carolina 27599 \\ ${ }^{c}$ Chinese Medicine Research and Development Center, China Medical University and Hospital, \\ Taichung, Taiwan
}

\begin{abstract}
In our continuing search for natural product-based spin-labeled antitumor drugs, 20 novel spinlabeled camptothecin derivatives were synthesized via a $\mathrm{Cu}$-catalyzed one pot reaction and evaluated for cytotoxicity against four human tumor cell lines (A-549, MDA-MB-231, KB, and KBvin). Eighteen of the target compounds (9a, 9b, 9d-9k, 9m-9t) exhibited significant in vitro antiproliferative activity against these four tested tumor cell lines. Compounds $9 \mathbf{e}$ and $\mathbf{9 j}\left(\mathrm{IC}_{50}\right.$ 0.057 and $0.072 \mu \mathrm{M}$, respectively) displayed the greatest cytotoxicity against the multidrugresistant (MDR) KBvin cell line and merit further development into preclinical and clinical drug candidates for treating cancer including MDR phenotype.
\end{abstract}

\section{Keywords}

Camptothecin; C-20 position; cytotoxic activity; spin-labeled

\section{Introduction}

Camptothecin (CPT, 1, Figure 1), a pentacyclic alkaloid isolated from Camptotheca acuminata by Wall et al., showed excellent antitumor activity against a broad spectrum of tumor cell lines by inhibiting topoisomerase I (Topo I). ${ }^{1-5}$ Two semisynthetic derivatives, topotecan (2) and irinotecan (3), are widely used clinically for treating ovarian and smallcell lung cancers, respectively. Several other drug candidates, such as gimatecan (4),

(C) 2014 Elsevier Ltd. All rights reserved.

*To whom correspondence should be addressed. Tel.: (919) 962-0066. Fax: (919) 966-3893. khlee@ email.unc.edu (K.H. Lee); yqliu@lzu.edu.cn (Y.Q. Liu).

These authors contributed equally to this work.

Publisher's Disclaimer: This is a PDF file of an unedited manuscript that has been accepted for publication. As a service to our customers we are providing this early version of the manuscript. The manuscript will undergo copyediting, typesetting, and review of the resulting proof before it is published in its final citable form. Please note that during the production process errors may be discovered which could affect the content, and all legal disclaimers that apply to the journal pertain. 
CKD-602 (5), and BNP-1350 (6), are the subject of ongoing preclinical or clinical evaluation. ${ }^{6-9}$

Although CPT analogues remain a promising class of antitumor agents, their therapeutic use has been severely hindered by toxicity issues and delivery problems, due to poor water solubility, as well as intrinsic instability of the highly electrophilic a-hydroxylactone of the E ring, due to preferential binding of the opened carboxylate to serum albumin. ${ }^{10,11}$ This chemical feature diminished efficacy of various CPT derivatives in vivo compared to the spectacular results often obtained from in vitro studies and xenograft models. Thus, several promising strategies to overcome this challenge have been developed. These approaches include the development of prodrugs (conjugates and polymer bound CPTs), new formulations (liposomes or microparticulate carriers), and synthetic lipophilic CPTs. ${ }^{12-14}$ Most of these strategies aimed to maintain the active closed-lactone form in the plasma compartment, and encouraging results have been obtained. Additionally, extensive structureactivity relationship (SAR) investigations have suggested that the intact lactone ring $\mathrm{E}$ of CPT is the most critical structural feature with respect to antitumor activity. A free 20hydroxy group favors lactone ring-opening due to formation of intra-molecular hydrogen bonding, while acylation of this group should render the lactone moiety more stable toward ring opening. ${ }^{15,16}$ Many studies have been focused on highly efficient semisynthetic methodologies paving the way for development of new potent C-20-modified CPT analogues. Indeed, our own results, ${ }^{17,18,19}$ as well as those of others with 20(S)-O-acyl esters, ${ }^{20,21} 20(S)$-O-carbonate linked tripeptide conjugates, ${ }^{22}$ and $20(S)$ - $O$-linked glycoconjugates, ${ }^{23}$ have supported the importance of esterified CPT derivatives for potent cytotoxic and antitumor activity. Esterification of the 20-hydroxyl group also enhances plasma stability compared with unmodified CPT, as well as augments in vivo superior antitumor activity without notable toxicities in liver, lung, kidney, and spleen. ${ }^{19}$

Furthermore, novel nitroxide-derived spin-labeling of antitumor drugs is a promising direction in anticancer chemotherapy, not only because these compounds exhibit superior cytotoxic activity, but also because they can be monitored by electron paramagnetic resonance (EPR) in pharmacological experiments. Based on current knowledge, the introduction of a stable nitroxyl radical into pharmaceutical molecules can reduce toxicity and potentiate antitumor effects to a certain degree. Some studies have shown that the introduction of a nitroxyl moiety can lead to fast decomposition, higher alkylating and lower carbamoylating activity, better anti-melanomic activity, lower general toxicity, and the ability to transport molecules through cell membranes, while the nitroxyl free radicals themselves possess low toxicity and are not mutagenic or carcinogenic. ${ }^{24-28}$ In our prior studies, we successfully prepared a number of spin-labeled derivatives of known antitumor agents, such as podophyllotoxin, ${ }^{29-36} \mathrm{CPT},{ }^{37}$ rotenone, ${ }^{38}$ glycyrrhetinic acid, ${ }^{39}$ and combretastatin, ${ }^{40}$ resulting in compounds with superior pharmacological properties compared to those of the parent compounds. Inspired by this prior work, we herein report the design, synthesis, and in vitro cytotoxicity of a series of novel 20-modified spin-labeled CPT derivatives as part of our continuing search for promising natural product-derived anticancer agents. 


\section{Results and discussion}

\subsection{Chemistry}

The synthetic routes to target compounds are outlined in Scheme 1. Briefly, the 20-hydroxyl of CPT was esterified with various $N$-Boc-amino acid derivatives (7) in suitable yields by a simple modification of the carbodiimide method using a combination of $N, N^{\prime}$ diisopropylcarbodiimide (DIPC) and 4-dimethylaminopyridine (DMAP). Removal of the $\mathrm{N}$ Boc group of 7 with trifluoroacetic acid (TFA) in $\mathrm{CH}_{2} \mathrm{Cl}_{2}$ (1:1) formed the key intermediate TFA salts 8 . Subsequently, these key precursors were successfully combined with sulfonylazides and alkynes in a $\mathrm{Cu}$-catalyzed three-component reaction ${ }^{41}$ to afford the target compounds 9a-t in 59-75\% yields. All newly synthesized compounds were purified by column chromatography and their structures were characterized by ESI-MS, EPR, IR, and elemental analysis.

\subsection{Cytotoxicity}

Target compounds 9a-t were evaluated against a panel of human tumor cell lines, including A-549 (lung carcinoma), MDA-MB-231 (triple-negative breast cancer), KB (nasopharyngeal carcinoma), and KBvin (vincristine resistant KB subline), using a sulforhodamine B colorimetric (SRB) assay with triplicate experiments. Topotecan (2) was used as a positive control and the antiproliferative activities of compounds are shown in Table 1. Except for compounds $\mathbf{9 c}$ and $\mathbf{9 l}$, all target compounds exhibited significant in vitro antiproliferative activity against the four tested tumor cell lines, with $\mathrm{IC}_{50}$ values ranging from 0.055 to $0.84 \mu \mathrm{M}$, and were as or more potent than 2 . Remarkably, all of the compounds were more potent than $2\left(\mathrm{IC}_{50}, 0.40 \mu \mathrm{M}\right)$ against the multidrug-resistant KBvin cell line, with $9 \mathbf{e}\left(\mathrm{IC}_{50}, 0.057 \mu \mathrm{M}\right)$ and $\mathbf{9 j}\left(\mathrm{IC}_{50}, 0.072 \mu \mathrm{M}\right)$ showing the greatest cytotoxicity against this cell line. Thus, spin-labeling of $\mathrm{CPT}$ at the $\mathrm{C}$-20-hydroxyl position might overcome the MDR phenotype. As commonly found with prodrugs, esterification of the C-20-hydroxyl of CPT with different sulfonylamidine side chains led to somewhat decreased cytotoxic activity against A549, DU-145, and KB tumor cell lines in comparison to $2 .{ }^{19}$ This result is in agreement with our prior observations with C-20-substituted CPT derivatives as potent prodrugs. ${ }^{37} \mathrm{The}^{\mathrm{IC}_{50}}$ values also revealed that the A-549 cell line was more sensitive than the other three cell lines to these compounds, which is consistent with the clinical behavior of other CPT derivatives. ${ }^{20}$

Furthermore, some preliminary SAR correlations were also observed for these spin-labeled 20-sulfonylamidine CPT derivatives. As shown in Scheme 1, the modified groups included $\mathrm{R}_{1}$ on the amidine carbon, $\mathrm{R}_{2}$ on the sulfonyl moiety, and $\mathrm{R}_{3}$ as the nitroxide moiety. When the $R_{2}$ and $R_{3}$ substituents were kept constant and the $R_{1}$ group in the sulfonylamidine was varied, hydrogen (9a) and methyl (9b) gave the best results compared with the larger alkyl groups in 9c (isopropyl), 9k (isobutyl), and $9 \mathbf{l}$ (sec-butyl), suggesting that small aliphatic chains appear to be the best $\mathrm{R}_{1}$ substituents for greater cytotoxic potency. Moreover, when the $R_{1}$ group was fixed as hydrogen, $R_{3}$ was fixed as a piperidinyl moiety, and the $R_{2}$ group in the sulfonylamidine was varied, similar results were seen in the corresponding derivatives 9d-j, 9n, and 9o, indicating that the substituent's size is critical. Furthermore, the cytotoxicity of these compounds was distinctly correlated with the nitroxide moiety. Also, 
the ring size and degree of unsaturation did not obviously affect the potency of the target compounds against three (A-549, MDA-MB-231, KB) of the four tested tumor cell lines, which was consistent with the literature. ${ }^{32}$ Overall, the results suggest that the cytotoxic potencies of our designed derivatives were dual controlled by altering the length of the sulfonylamidine arm as well as the size of the substituent group. The best antiproliferative activity was achieved only with an appropriate balance between flexibility and size, such as in $9 \mathbf{e}$ and $\mathbf{9 j}$.

\section{Conclusion}

In summary, application of free radical compounds of the stable nitroxide type has become increasingly frequent in medicinal chemistry studies during recent years. In particular, design and synthesis of spin-labeled antitumor drugs using nitroxide groups as biological response modifiers have elicited widespread interest in cancer chemotherapy. As an extension to our studies on spin-labeled antitumor drugs, we designed and synthesized a series of novel 20-modified spin-labeled CPT derivatives, which were then evaluated for antiproliferative activities against four tumor cell lines (A-549, MDA-MB-231, KB, KBvin) by using a sulforhodamine B colorimetric assay. The cytotoxic results showed that most of the new spin-labeled compounds exhibited significant antiproliferative activities against four tumor cell lines. Notably, except for compounds $9 \mathbf{c}, 9 \mathbf{k}$, and $9 \mathbf{l}$, all compounds were more potent than 2 against multidrug-resistant KBvin cells. SAR analysis indicated that the size, electron density, and distribution of the substituents within the sulfonylamidine side chain are critical to the derivatives' activity. These findings support our further optimization of CPT to develop potential spin-labeled CPT-derived anticancer drug candidates. Continuing studies to substantiate and improve activity profiles are underway in our laboratory and will be reported in due course.

\section{Experimental section}

\subsection{General}

All reagents and solvents were of reagent grade or purified according to standard methods before use. Analytical thin-layer chromatography (TLC) and preparative thin-layer chromatography (PTLC) were performed with silica gel plates using silica gel 60 GF254 (Qingdao Haiyang Chemical Co., Ltd.). Melting points were taken on a Kofler melting point apparatus and are uncorrected. IR spectra were measured on a Nicolet 380 FT-IR spectrometer on neat samples placed between $\mathrm{KBr}$ plates. The EPR spectra were obtained with a Bruker A300 X-band EPR spectrometer. MS analyses were performed on ZAB-HS and Bruker Daltonics APEXII49e instruments. The starting CPT was isolated from the Chinese medicinal plant $C$. acuminata, and was purified before being used. ${ }^{3}$ The intermediate CPT-20-esters of $N$-Boc-amino acid derivatives $\mathbf{7}$ and their TFA salts 8 were synthesized according to our previous procedures. ${ }^{37}$

\subsection{General synthetic procedure for compounds $9 a-9 t$}

Triethylamine $(1.2 \mathrm{mmol})$ was added slowly to a suspension of the various TFA salts $\mathbf{8}(0.5$ $\mathrm{mmol})$ in $\mathrm{CH}_{2} \mathrm{Cl}_{2}(35 \mathrm{~mL})$, and this mixture was stirred for $10 \mathrm{~min}$ when a clear solution was obtained. Under an $\mathrm{N}_{2}$ atmosphere, alkyne $(0.5 \mathrm{mmol})$, sulfonylazide $(0.6 \mathrm{mmol})$, and $\mathrm{CuI}$ 
$(0.05 \mathrm{mmol})$ were added into this reaction mixture at room temperature. After the reaction was completed, as monitored by TLC, the reaction mixture was diluted by adding $\mathrm{CH}_{2} \mathrm{Cl}_{2}$ (4 $\mathrm{mL}$ ) and aqueous $\mathrm{NH}_{4} \mathrm{Cl}$ solution $(6 \mathrm{~mL})$. The mixture was stirred for an additional $30 \mathrm{~min}$ and two layers were separated. The aqueous layer was extracted with $\mathrm{CH}_{2} \mathrm{Cl}_{2}(3 \mathrm{~mL} \times 3)$. The combined organic layers were dried over $\mathrm{MgSO}_{4}$, filtered, and concentrated in vacuo. The crude residue was purified by flash column chromatography with an appropriate eluting solvent system.

Compound 9a. Yield 70\%; yellow orange solid; mp 133-134 ${ }^{\circ} \mathrm{C}$; IR $(\mathrm{KBr}) \vee\left(\mathrm{cm}^{-1}\right)$ : 3432 , 2978, 2939, 1753, 1664, 1618, 1560, 1449, 1402, 1385 (NO), 1273, 1147, 1094, 881, 761, 669, 616, 556; Anal. Calcd for $\mathrm{C}_{41} \mathrm{H}_{46} \mathrm{~N}_{5} \mathrm{O}_{9} \mathrm{~S}: \mathrm{C}, 62.74 ; \mathrm{H}, 5.91 ; \mathrm{N}, 8.92$. Found: C, 62.70; $\mathrm{H}, 6.01$; N, 8.87; EPR: $\mathrm{g}_{0}=2.0064, A \mathrm{n}=14.62 \mathrm{Gs}$ (triplet peak in $1 \times 10^{-4} \mathrm{M}, \mathrm{DMF}$ ); MS-ESI $m / z: 785.3[\mathrm{M}+\mathrm{H}]^{+}$.

Compound 9b. Yield 67\%; yellow orange solid; mp 118-121 ${ }^{\circ} \mathrm{C}$; IR $(\mathrm{KBr}) \vee\left(\mathrm{cm}^{-1}\right)$ : 3433, 2977, 2938, 1752, 1664, 1618, 1545, 1458, 1403, 1384 (NO), 1273, 1145, 1090, 881, 787, 724, 616, 555; Anal. Calcd for $\mathrm{C}_{42} \mathrm{H}_{48} \mathrm{~N}_{5} \mathrm{O}_{9} \mathrm{~S}: \mathrm{C}, 63.14 ; \mathrm{H}, 6.06$; N, 8.77. Found: C, 63.10; $\mathrm{H}, 6.01$; N, 8.90; EPR: $\mathrm{g}_{0}=2.0060, A \mathrm{n}=14.62 \mathrm{Gs}$ (triplet peak in $1 \times 10^{-4} \mathrm{M}, \mathrm{DMF}$ ); MS-ESI $m / z: 800.2[\mathrm{M}+2 \mathrm{H}]^{+}$.

Compound 9c. Yield 71\%; yellow orange solid; mp $128-130{ }^{\circ} \mathrm{C}$; IR $(\mathrm{KBr}) v\left(\mathrm{~cm}^{-1}\right)$ : 3448 , 2975, 2930, 2873, 1751, 1666, 1618, 1561, 1546, 1439, 1402, 1385 (NO), 1272, 1145 , 1091, 881, 670, 616, 555; Anal. Calcd for $\mathrm{C}_{44} \mathrm{H}_{52} \mathrm{~N}_{5} \mathrm{O}_{9} \mathrm{~S}: \mathrm{C}, 63.90 ; \mathrm{H}, 6.34 ; \mathrm{N}, 8.47$. Found: C, 64.03; H, 6.31; N, 8.56; EPR: $\mathrm{g}_{0}=2.0061, A \mathrm{n}=14.62 \mathrm{Gs}$ (triplet peak in $1 \times 10^{-4} \mathrm{M}, \mathrm{DMF}$ ); MS-ESI $m / z: 828.3[\mathrm{M}+2 \mathrm{H}]^{+}$.

Compound 9d. Yield 70\%; yellow orange solid; mp 125-127 ${ }^{\circ} \mathrm{C}$; IR $(\mathrm{KBr}) \vee\left(\mathrm{cm}^{-1}\right)$ : 3448, 2974, 2929, 1752, 1655, 1618, 1560, 1508, 1402, 1385 (NO) 1272, 1122, 881, 788, 670, 617, 474; Anal. Calcd for $\mathrm{C}_{35} \mathrm{H}_{42} \mathrm{~N}_{5} \mathrm{O}_{9} \mathrm{~S}: \mathrm{C}, 59.31 ; \mathrm{H}, 5.97 ; \mathrm{N}, 9.88$. Found: C, 59.43; H, 6.01; N, 8.79; EPR: $\mathrm{g}_{0}=2.0066, A \mathrm{n}=14.62 \mathrm{Gs}$ (triplet peak in $1 \times 10^{-4} \mathrm{M}, \mathrm{DMF}$ ); MS-ESI $\mathrm{m} / z$ : $731.3[\mathrm{M}+\mathrm{Na}]^{+}$.

Compound 9e. Yield 64\%; yellow orange solid; mp 124-126 ${ }^{\circ} \mathrm{C}$; IR $(\mathrm{KBr}) v\left(\mathrm{~cm}^{-1}\right)$ : 3431 , 2974, 2929, 1753, 1655, 1618, 1561, 1508, 1439, 1403, 1384 (NO) ) 1270, 1233, 1118, 881, 787, 758, 616, 475; Anal. Calcd for $\mathrm{C}_{36} \mathrm{H}_{44} \mathrm{~N}_{5} \mathrm{O}_{9} \mathrm{~S}: \mathrm{C}, 59.82 ; \mathrm{H}, 6.14 ; \mathrm{N}, 9.69$. Found: C, 59.73; H, 6.08; N, 9.58; EPR: $\mathrm{g}_{0}=2.0064, A \mathrm{n}=14.62 \mathrm{Gs}$ (triplet peak in $1 \times 10^{-4} \mathrm{M}, \mathrm{DMF}$ ); MS-ESI $m / z: 745.4[\mathrm{M}+\mathrm{Na}]^{+}$.

Compound 9f. Yield 66\%; yellow orange solid; mp 123-125 ${ }^{\circ} \mathrm{C}$; IR (KBr) v (cm $\left.{ }^{-1}\right)$ : 3423 , 2973, 2926, 2870, 1758, 1663, 1618, 1561, 1458, 1404, 1384 (NO), 1267, 1234, 1181, 1119, 881, 788, 763, 724, 669, 616; Anal. Calcd for $\mathrm{C}_{38} \mathrm{H}_{48} \mathrm{~N}_{5} \mathrm{O}_{9} \mathrm{~S}$ : C, 60.78; H, 6.44; N, 9.33. Found: C, 60.89; H, 6.38; N, 9.50; EPR: $\mathrm{g}_{0}=2.0060, A \mathrm{n}=14.62 \mathrm{Gs}$ (triplet peak in $\left.1 \times 10^{-4} \mathrm{M}, \mathrm{DMF}\right) ; \mathrm{MS}-\mathrm{ESI} \mathrm{m} / \mathrm{z}: 773.4[\mathrm{M}+\mathrm{Na}]^{+}$.

Compound 9g. Yield 68\%; yellow orange solid; mp 142-144 ${ }^{\circ} \mathrm{C}$; IR $(\mathrm{KBr}) \vee\left(\mathrm{cm}^{-1}\right)$ : 3423, 2975, 2931, 1753, 1664, 1618, 1560, 1499, 1458, 1403, 1384 (NO) ) 1257, 1146, 1094, 881, 854, 805, 762, 724, 696, 614, 555; Anal. Calcd for $\mathrm{C}_{41} \mathrm{H}_{46} \mathrm{~N}_{5} \mathrm{O}_{10} \mathrm{~S}$ : C, 61.49; H, 5.79; N, 
8.74. Found: C, 61.54; H, 5.86; N, 8.67; EPR: $\mathrm{g}_{0}=2.0062, A \mathrm{n}=14.62 \mathrm{Gs}$ (triplet peak in $\left.1 \times 10^{-4} \mathrm{M}, \mathrm{DMF}\right) ; \mathrm{MS}-\mathrm{ESI} \mathrm{m} / \mathrm{z}: 823.2[\mathrm{M}+\mathrm{Na}]^{+}$.

Compound 9h. Yield 73\%; yellow orange solid; mp 144-146 ${ }^{\circ} \mathrm{C}$; IR $(\mathrm{KBr}) \vee\left(\mathrm{cm}^{-1}\right)$ : 3423 , 2975, 2934, 1753, 1666, 1618, 1561, 1508, 1458, 1402, 1385 (NO), 1272, 1234, 1149, 1095, 881, 762, 726, 618, 483; Anal. Calcd for $\mathrm{C}_{40} \mathrm{H}_{43} \mathrm{ClN}_{5} \mathrm{O}_{9} \mathrm{~S}$ : C, 59.66; H, 5.38; N, 8.70. Found: C, 59.70; $\mathrm{H}, 5.45 ; \mathrm{N}, 8.57$; EPR: $\mathrm{g}_{0}=2.0064, \mathrm{An}=14.62 \mathrm{Gs}$ (triplet peak in $1 \times 10^{-4} \mathrm{M}$, DMF); MS-ESI $m / z: 806.3[\mathrm{M}+2 \mathrm{H}]^{+}$.

Compound 9i. Yield 64\%; yellow orange solid; mp 105-107 ${ }^{\circ} \mathrm{C}$; IR $(\mathrm{KBr}) \vee\left(\mathrm{cm}^{-1}\right)$ : 3422, 2975, 2930, 2871, 1753, 1663, 1593, 1560, 1496, 1458, 1403, 1383 (NO), 1272, 1236, 1147, 1091, 1050, 880, 839, 764, 669, 616, 555; Anal. Calcd for $\mathrm{C}_{40} \mathrm{H}_{43} \mathrm{FN}_{5} \mathrm{O}_{9} \mathrm{~S}: \mathrm{C}, 60.90$; H, 5.49; N, 8.88. Found: C, 61.01; H, 5.55; N, 8.49; EPR: $\mathrm{g}_{0}=2.0066, A \mathrm{n}=14.62 \mathrm{Gs}$ (triplet peak in $\left.1 \times 10^{-4} \mathrm{M}, \mathrm{DMF}\right)$; MS-ESI $\mathrm{m} / z: 790.3[\mathrm{M}+2 \mathrm{H}]^{+}$.

Compound 9j. Yield 68\%; yellow orange solid; mp 135-137 ${ }^{\circ} \mathrm{C}$; IR $(\mathrm{KBr}) \vee\left(\mathrm{cm}^{-1}\right)$ : 3393, 2973, 2901, 1757, 1663, 1606, 1560, 1529, 1450, 1404, 1384 (NO), 1351, 1223, 1150, 1081, 1050, 880, 747, 617, 464; Anal. Calcd for $\mathrm{C}_{40} \mathrm{H}_{43} \mathrm{~N}_{6} \mathrm{O}_{11} \mathrm{~S}: \mathrm{C}, 58.89 ; \mathrm{H}, 5.31$; , 10.30. Found: C, 58.94; H, 5.45; N, 10.25; EPR: $\mathrm{g}_{0}=2.0060, A \mathrm{n}=14.62 \mathrm{Gs}$ (triplet peak in $\left.1 \times 10^{-4} \mathrm{M}, \mathrm{DMF}\right) ; \mathrm{MS}-\mathrm{ESI} \mathrm{m} / z: 817.4[\mathrm{M}+2 \mathrm{H}]^{+}$.

Compound 9k. Yield 62\%; yellow orange solid; mp 120-122 ${ }^{\circ} \mathrm{C}$; IR $(\mathrm{KBr}) \vee\left(\mathrm{cm}^{-1}\right): 3385$, 2973, 2900, 1750, 1663, 1618, 1549, 1452, 1404, 1384 (NO), 1272, 1234, 1146, 1087, 1050, 880, 814, 763, 689, 618, 556; Anal. Calcd for $\mathrm{C}_{45} \mathrm{H}_{54} \mathrm{~N}_{5} \mathrm{O}_{9} \mathrm{~S}: \mathrm{C}, 64.27 ; \mathrm{H}, 6.47$; N, 8.33. Found: C, 64.38; H, 6.56; N, 8.21; EPR: $\mathrm{g}_{0}=2.0062, A \mathrm{n}=14.62 \mathrm{Gs}$ (triplet peak in $\left.1 \times 10^{-4} \mathrm{M}, \mathrm{DMF}\right) ; \mathrm{MS}-\mathrm{ESI} \mathrm{m} / \mathrm{z}: 842.4[\mathrm{M}+2 \mathrm{H}]^{+}$.

Compound 91. Yield 70\%; yellow orange solid; mp 122-124 ${ }^{\circ} \mathrm{C}$; IR $(\mathrm{KBr}) \vee\left(\mathrm{cm}^{-1}\right)$ : 3401, 2973, 2935, 2873, 1751, 1664, 1604, 1545, 1458, 1403, 1384 (NO), 1273, 1147, 1091, 1051, 881, 815, 669, 617, 556; Anal. Calcd for $\mathrm{C}_{45} \mathrm{H}_{54} \mathrm{~N}_{5} \mathrm{O}_{9} \mathrm{~S}$ : C, 64.27; H, 6.47; N, 8.33. Found: $\mathrm{C}, 64.38 ; \mathrm{H}, 6.56 ; \mathrm{N}, 8.21$; EPR: $\mathrm{g}_{0}=2.0064, \mathrm{An}=14.62 \mathrm{Gs}$ (triplet peak in $1 \times 10^{-4} \mathrm{M}$, DMF); MS-ESI $m / z: 842.4[\mathrm{M}+2 \mathrm{H}]^{+}$.

Compound 9m. Yield 68\%; yellow orange solid; mp 112-114 ${ }^{\circ} \mathrm{C}$; IR $(\mathrm{KBr}) \vee\left(\mathrm{cm}^{-1}\right)$ : 3402, 2974, 2901, 1751, 1655, 1618, 1560, 1544, 1450, 1405, 1384 (NO), 1250, 1079, 1066, 1050, 881, 701, 617; Anal. Calcd for $\mathrm{C}_{48} \mathrm{H}_{52} \mathrm{~N}_{5} \mathrm{O}_{9} \mathrm{~S}: \mathrm{C}, 65.89 ; \mathrm{H}, 5.99 ; \mathrm{N}, 8.00$. Found: C, 65.98; H, 6.12; N, 8.06; EPR: $\mathrm{g}_{0}=2.0066, A \mathrm{n}=14.62 \mathrm{Gs}$ (triplet peak in $1 \times 10^{-4} \mathrm{M}, \mathrm{DMF}$ ); MS-ESI $m / z: 876.4[\mathrm{M}+2 \mathrm{H}]^{+}$.

Compound 9n. Yield 59\%; yellow orange solid; $\mathrm{mp} 141-143{ }^{\circ} \mathrm{C}$; IR $(\mathrm{KBr}) \vee\left(\mathrm{cm}^{-1}\right)$ : 3411 , 2974, 2932, 1754, 1662, 1604, 1560, 1458, 1403, 1384 (NO), 1272, 1236, 1127,1082, 1050, 880, 787, 724, 688, 619, 555, 480; Anal. Calcd for $\mathrm{C}_{44} \mathrm{H}_{46} \mathrm{~N}_{5} \mathrm{O}_{9} \mathrm{~S}: \mathrm{C}, 64.37 ; \mathrm{H}, 5.65 ; \mathrm{N}$, 8.53. Found: C, 64.30; H, 5.54; N, 8.46; EPR: $\mathrm{g}_{0}=2.0062, A \mathrm{n}=14.62 \mathrm{Gs}$ (triplet peak in $\left.1 \times 10^{-4} \mathrm{M}, \mathrm{DMF}\right) ;$ MS-ESI $\mathrm{m} / \mathrm{z}: 822.4[\mathrm{M}+2 \mathrm{H}]^{+}$.

Compound 9o. Yield 67\%; yellow orange solid; mp 136-138 ${ }^{\circ} \mathrm{C}$; IR $(\mathrm{KBr}) \vee\left(\mathrm{cm}^{-1}\right): 3423$, 2975, 2938, 1753, 1663, 1617, 1560, 1458, 1404, 1384 (NO), 1292, 1234, 1143, 1093, 
1015, 881, 762, 724, 669, 618, 592; Anal. Calcd for $\mathrm{C}_{38} \mathrm{H}_{42} \mathrm{~N}_{5} \mathrm{O}_{9} \mathrm{~S}_{2}$ : C, 58.75; H, 5.45; N, 9.01. Found: C, 58.65; H, 5.38; N, 9.21; EPR: $\mathrm{g}_{0}=2.0066, A \mathrm{n}=14.62 \mathrm{Gs}$ (triplet peak in $\left.1 \times 10^{-4} \mathrm{M}, \mathrm{DMF}\right) ;$ MS-ESI $m / z: 878.3[\mathrm{M}+2 \mathrm{H}]^{+}$.

Compound 9p. Yield 75\%; yellow orange solid; mp 105-107 ${ }^{\circ} \mathrm{C}$; IR $(\mathrm{KBr}) \vee\left(\mathrm{cm}^{-1}\right)$ : 3423, 2974, 2928, 1756, 1663, 1601, 1560, 1457, 1403, 1384 (NO), 1273, 1236, 1177, 1147, 1094, 1050, 920, 881, 818, 763, 695, 615, 556, 480; Anal. Calcd for $\mathrm{C}_{42} \mathrm{H}_{48} \mathrm{~N}_{5} \mathrm{O}_{9} \mathrm{~S}: \mathrm{C}$, 63.14; H, 6.06; N, 8.77. Found: C, 63.04; H, 6.19; N, 8.92; EPR: $\mathrm{g}_{0}=2.0060, A \mathrm{n}=14.62 \mathrm{Gs}$ (triplet peak in $1 \times 10^{-4} \mathrm{M}, \mathrm{DMF}$ ); MS-ESI $m / z: 800.4[\mathrm{M}+2 \mathrm{H}]^{+}$.

Compound 9q. Yield 65\%; light yellow solid; mp 121-123 ${ }^{\circ} \mathrm{C}$; IR $(\mathrm{KBr}) \vee\left(\mathrm{cm}^{-1}\right)$ : 3415 , 2973, 2931, 2871, 1757, 1663, 1603, 1561, 1442, 1402, 1384 (NO), 1256, 1190, 1120, 1084, 1049, 977, 881, 787, 724, 613, 562, 475; Anal. Calcd for $\mathrm{C}_{35} \mathrm{H}_{40} \mathrm{~N}_{5} \mathrm{O}_{9} \mathrm{~S}: \mathrm{C}, 59.48 ; \mathrm{H}$, 5.70; N, 9.91. Found: C, 59.56; H, 5.63; N, 10.02; EPR: $\mathrm{g}_{0}=2.0058, A \mathrm{n}=14.76 \mathrm{Gs}$ (triplet peak in $1 \times 10^{-4} \mathrm{M}$, DMF); MS-ESI $\mathrm{m} / z: 729.3[\mathrm{M}+\mathrm{Na}]^{+}$;

Compound 9r. Yield 61\%; light yellow solid; mp 110-112 ${ }^{\circ} \mathrm{C}$; IR $(\mathrm{KBr}) v\left(\mathrm{~cm}^{-1}\right): 3422$, 2974, 2929, 1752, 1655, 1603, 1560, 1458, 1439, 1384 (NO), 1273, 1147, 1093, 1050, 881, 724, 695, 616, 556; Anal. Calcd for $\mathrm{C}_{41} \mathrm{H}_{44} \mathrm{~N}_{5} \mathrm{O}_{9} \mathrm{~S}$ : C, 62.90; H, 5.66; N, 8.95. Found: C, 63.00; H, 5.53; N, 9.01; EPR: $\mathrm{g}_{0}=2.0055, A \mathrm{n}=14.76 \mathrm{Gs}$ (triplet peak in $1 \times 10^{-4} \mathrm{M}, \mathrm{DMF}$ ); MS-ESI $m / z: 805.4[\mathrm{M}+\mathrm{Na}]^{+}$.

Compound 9s. Yield 70\%; light yellow solid; mp 120-122 ${ }^{\circ} \mathrm{C}$; IR $(\mathrm{KBr}) \vee\left(\mathrm{cm}^{-1}\right)$ : 3415 , 2974, 2929, 1756, 1664, 1598, 1560, 1499, 1458, 1403, 1384 (NO), 1258, 1146, 1094, 1051, 881, 836, 805, 725, 697, 614, 567; Anal. Calcd for $\mathrm{C}_{41} \mathrm{H}_{44} \mathrm{~N}_{5} \mathrm{O}_{10} \mathrm{~S}: \mathrm{C}, 61.64$; H, 5.55; $\mathrm{N}$, 8.77. Found: C, 61.81; H, 5.42; N, 8.85; EPR: $\mathrm{g}_{0}=2.0058, A \mathrm{n}=14.76 \mathrm{Gs}$ (triplet peak in $\left.1 \times 10^{-4} \mathrm{M}, \mathrm{DMF}\right) ; \mathrm{MS}-\mathrm{ESI} \mathrm{m} / z: 800.3[\mathrm{M}+2 \mathrm{H}]^{+}$.

Compound 9t. Yield 69\%; light yellow solid; mp 103-105 ${ }^{\circ} \mathrm{C}$; IR $(\mathrm{KBr}) \vee\left(\mathrm{cm}^{-1}\right)$ : 3423, 2974, 2927, 1753, 1663, 1592, 1560, 1495, 1458, 1403, 1384 (NO), 1279, 1234, 1186, 1148, 1091, 880, 839, 763, 724, 699, 613, 554, 481; Anal. Calcd for $\mathrm{C}_{40} \mathrm{H}_{41} \mathrm{FN}_{5} \mathrm{O}_{9} \mathrm{~S}: \mathrm{C}$, 61.06; H, 5.25; N, 8.90. Found: C, 61.21; H, 5.15; N, 9.02; EPR: $\mathrm{g}_{0}=2.0055, A \mathrm{n}=14.76 \mathrm{Gs}$ (triplet peak in $1 \times 10^{-4} \mathrm{M}, \mathrm{DMF}$ ); MS-ESI $\mathrm{m} / \mathrm{z}: 809.4[\mathrm{M}+\mathrm{Na}]^{+}$.

\subsection{Cytotoxicity assays}

The human tumor cell lines, A549 (lung carcinoma), MDA-MB-231 (triple-negative breast cancer), KB (originally isolated from epidermoid carcinoma of the nasopharynx), and KBvin (vincristine-resistant $\mathrm{KB}$ subline), were obtained from the Lineberger Comprehensive Cancer Center (UNC-CH) or from ATCC (Manassas, VA), except KBvin, which was a generous gift of Professor Y.-C. Cheng (Yale University). All cell lines were maintained and assayed in RPMI-1640 medium containing $2 \mathrm{mM}$ L-glutamine and $25 \mathrm{mM}$ HEPES (HyClone), supplemented with 10\% heat-inactivated fetal bovine serum (HyClone), 100 $\mu \mathrm{g} / \mathrm{mL}$ streptomycin, $100 \mathrm{IU} / \mathrm{mL}$ penicillin, and $0.25 \mu \mathrm{g} / \mathrm{mL}$ amphotericin B (Cellgro) in a humidified atmosphere containing $5 \% \mathrm{CO}_{2}$ in air. Cytotoxic activity was determined by the sulforhodamine B (SRB) colorimetric assay as previously described. ${ }^{37}$ In brief, the cells $\left(4.0-7.5 \times 10^{3}\right.$ cells/well) were seeded in 96-well plates filled with various concentrations of 
samples in culture medium, and cultured for $72 \mathrm{~h}$ at $37{ }^{\circ} \mathrm{C}$ with $5 \% \mathrm{CO}_{2}$ in air. At the end of the exposure period, the surviving cells were fixed in $10 \%$ trichloroacetic acid for $30 \mathrm{~min}$ at room temperature followed by staining with $0.04 \%$ SRB (Sigma Chemical Co.) for $30 \mathrm{~min}$. The protein-bound SRB was solubilized with $10 \mathrm{mM}$ Tris-base and the absorbance of SRB was measured at $515 \mathrm{~nm}$ on a ELx800 Microplate Reader (Bio-Tek Instruments, Winooski, VT) operated by Gen5 software. All results were representative of three or more experiments and expressed as an average with standard deviation (SD).

\section{Acknowledgments}

This work was supported financially by the National Natural Science Foundation of China (30800720, 31371975); the Fundamental Research Funds for the Central Universities (lzujbky-2014-k19), as well as the Foundation of Priority Forestry Disciplines in Zhejiang A\&F University (KF201325). Support was also provided by NIH grant CA177584 from the National Cancer Institute awarded to K.H. Lee. Thanks are also due to the support of Taiwan Department of Health Cancer Research Center of Excellence (DOH-100-TD-C-111-005).

\section{References}

1. Oberlies NH, Kroll DJ. J Nat Prod. 2004; 67:129. [PubMed: 14987046]

2. Slichenmyer WJ, Rowinsky EK, Donehower RC, Kaufmann SH. J Nat Cancer Inst. 1993; 85:271. [PubMed: 8381186]

3. Wall ME, Wani MC, Cook CE, Palmer KH, McPhail AT, Sim GA. J Am Chem Soc. 1966; 88:3888.

4. Takimoto CH, Wright J, Arbuck SG. Biochim Biophy Acta. 1998; 1400:107.

5. Pommier Y. Nat Rev Cancer. 2006; 6:789. [PubMed: 16990856]

6. Liew ST, Yang LX. Curr Pharm Des. 2008; 14:1078. [PubMed: 18473856]

7. Lorence A, Nessler CL. Phytochem. 2004; 65:2735.

8. Thomas CJ, Rahier NJ, Hecht SM. Bioorg Med Chem. 2004; 12:1585. [PubMed: 15028252]

9. Lerchen HG. Drugs of the Future. 2002; 27:869.

10. Adams DJ. Curr Med Chem - Anti-Cancer Agents. 2005; 5:1.

11. Tobin PJ, Rivory LP. Drug Design Reviews - Online. 2004; 1:341.

12. Hatefi A, Amsden B. Pharm Res. 2002; 19:1389. [PubMed: 12425455]

13. Onishi H, Machida Y. Curr Drug Discov Technol. 2005; 2:169. [PubMed: 16472226]

14. Sirikantaramas S, Asano T, Sudo H, Yamazaki M, Saito K. Curr Pharm Biotechnol. 2007; 8:196. [PubMed: 17691988]

15. Fassberg J, Stella VJ. J Pharm Sci. 1992; 81:676. [PubMed: 1403703]

16. Zhao H, Lee C, Sai P, Choe YH, Boro M, Pendri A, Guan S, Greenwald RB. J Org Chem. 2000; 65:4601. [PubMed: 10959865]

17. Liu YQ, Tian X, Yang L, Zhan ZC. Eur J Med Chem. 2008; 43:2610. [PubMed: 18313176]

18. Yang L, Zhao CY, Liu YQ. J Braz Chem Soc. 2011; 22:308.

19. Wang MJ, Liu YQ, Chang LC, Wang CY, Zhao YL, Zhao XB, Qian K, Nan X, Yang L, Yang XM, Hung HY, Yang JS, Kuo DH, Goto M, Morris-Natschke SL, Pan SL, Teng CM, Kuo SC, Wu TS, Wu TC, Lee KH. J Med Chem. 2014; 57:6008. [PubMed: 25003995]

20. Yang LX, Pan X, Wang H. Bioorg Med Chem Lett. 2002; 12:1241. [PubMed: 11965362]

21. Cao Z, Harris N, Kozielski A, Vardeman D, Stehlin JS, Giovanella B. J Med Chem. 1998; 41:31. [PubMed: 9438019]

22. De Groot FMH, Busscher GF, Aben RWM, Scheeren HW. Bioorg Med Chem Lett. 2002; 12:2371. [PubMed: 12161136]

23. Lerchen HG, Von dem Bruch K. J Prakt Chem. 2000; 342:753.

24. Naik N, Braslau R. Tetrahedron. 1998; 54:99.

25. Soule BP, Hyodo F, Matsumoto K, Simone NL, Cook JA, Krishna MC, Mitchell JB. Free Radical Biol Med. 2007; 42:1632. [PubMed: 17462532] 
26. Raikov ZD, Atanasov AT, Raikova ET. Med Hypotheses. 2003; 60:387. [PubMed: 12581616]

27. Gadjeva VG. Int J Pharm. 2002; 247:39. [PubMed: 12429483]

28. Zheleva A, Stanilova S, Dobreva Z, Zhelev Z. Int J Pharm. 2001; 222:237. [PubMed: 11427354]

29. Wang YG, Tian X, Chen YZ. Acta Pharm Sin. 1988; 23:792.

30. Liu YQ, Yang L, Tian X. Curr Bioact Compds. 2007; 3:37.

31. Tian X, Wang YG, Yang MG, Chen YZ. Life Sci. 1997; 60:511. [PubMed: 9042385]

32. Jin Y, Chen SW, Tian X. Bioorg Med Chem. 2006; 14:3062. [PubMed: 16406792]

33. Tian X, Zhang FM, Li WG. Life Sci. 2002; 70:2433. [PubMed: 12150207]

34. Liu YQ, Tian X. Syn Commun. 2005; 35:2749.

35. Zhang ZW, Zhang JQ, Hui L, Chen SW, Tian X. Eur J Med Chem. 2010; 45:1673. [PubMed: 20071056]

36. Kou L, Wang MJ, Wang LT, Zhao XB, Nan X, Yang L, Liu YQ, Morris-Natschke SL, Lee KH. Eur J Med Chem. 2014; 75:282. [PubMed: 24553146]

37. Liu YQ, Tian X, Yang L, Zhan ZC. Eur J Med Chem. 2008; 43:2610. [PubMed: 18313176]

38. Liu YQ, Ohkoshi E, Li LH, Yang L, Lee KH. Bioorg Med Chem Lett. 2012; 22:920. [PubMed: 22204911]

39. Liu YQ, Qian KD, Wang CY, Chen CH, Yang XM, Lee KH. Bioorg Med Chem Lett. 2012; 22:7530. [PubMed: 23122524]

40. Liu YQ, Li XJ, Zhao CY, Nan X, Tian J, Morris-Natschke SL, Zhang ZJ, Yang XM, Yang L, Li LH, Zhou XW, Lee KH. Bioorg Med Chem. 2013; 21:1248. [PubMed: 23369687]

41. Bae I, Han H, Chang S. J Am Chem Soc. 2005; 127:2038. [PubMed: 15713069]

42. Skehan P, Storeng R, Scudiero D, Monks A, McMahon J, Vistica D, Warren JT, Bokesch H, Kenney S, Boyd MR. J Natl Cancer Inst. 1990; 82:1107. [PubMed: 2359136] 

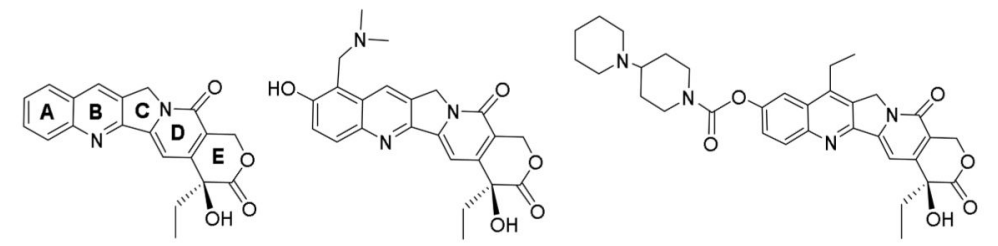

Camptothecin (1)

Topotecan (2)

Irinotecan (3)

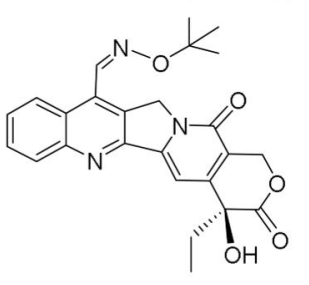

Gimatecan(4)

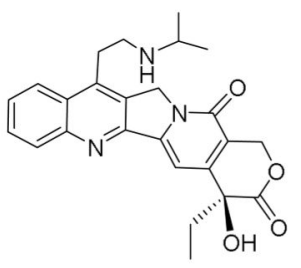

CKD-602 (5)

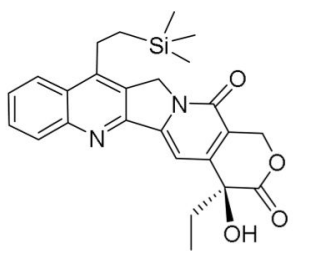

BNP-1350(6)

Figure 1.

Structures of camptothecin (1), topotecan (2), irinotecan (3), gimatecan (4), CKD-602 (5), and BNP-1350 (6). 

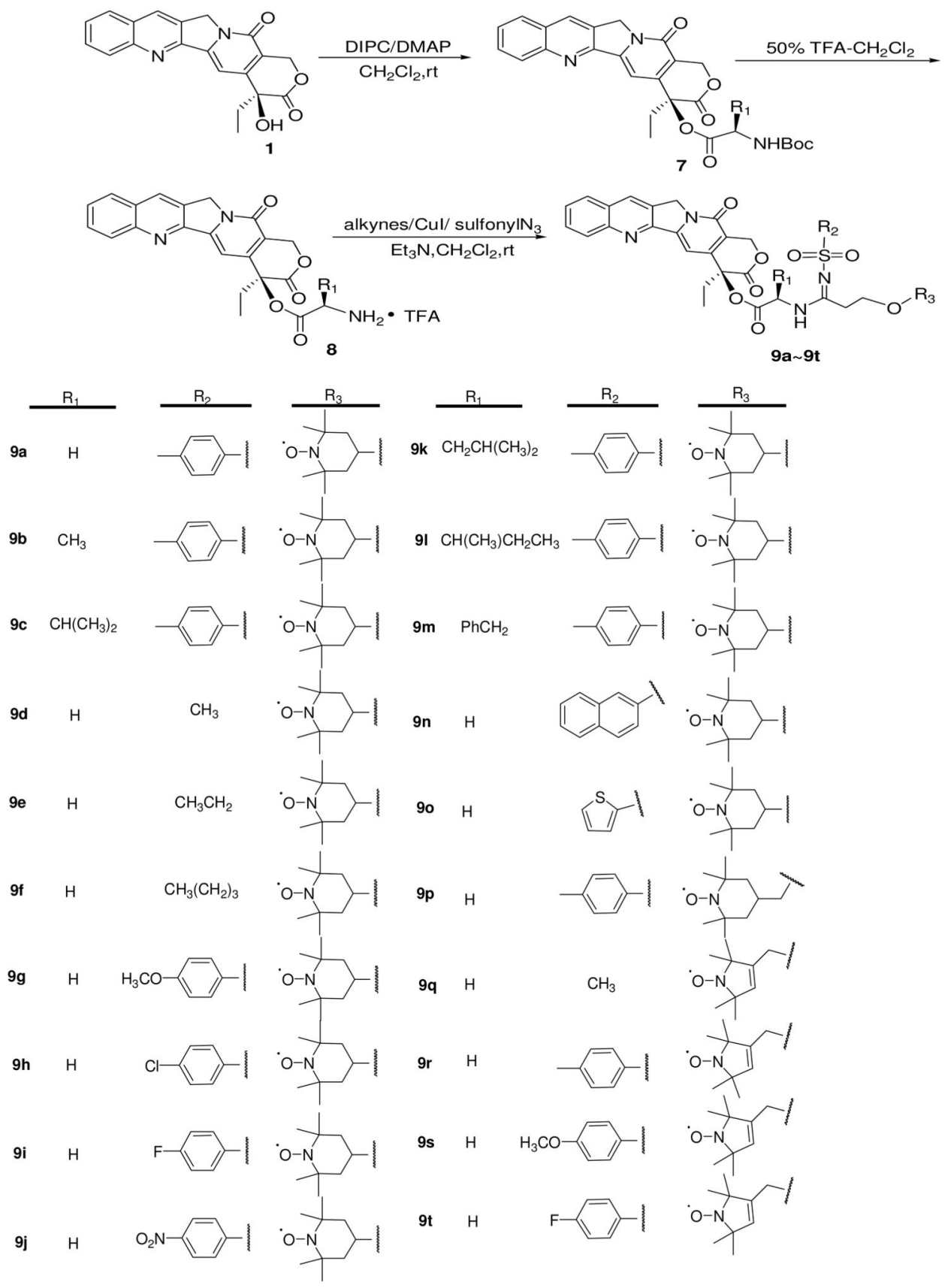

Scheme 1.

Synthesis of target compounds 9a-9t.

Bioorg Med Chem. Author manuscript; available in PMC 2015 November 15. 


\section{Table 1}

In vitro cytotoxicity of compounds $\mathbf{9 a - \mathbf { t }}$ against four human tumor cell lines. ${ }^{a}$

\begin{tabular}{|c|c|c|c|c|}
\hline \multirow{2}{*}{ Entry } & \multicolumn{4}{|c|}{$\mathrm{IC}_{50}(\mu \mathrm{M})(\text { Average } \pm \mathrm{SD})^{b}$} \\
\hline & A-549 & MDA-MB-231 & KB & KBvin \\
\hline $9 a$ & $0.076 \pm 0.026$ & $0.45 \pm 0.10$ & $0.21 \pm 0.016$ & $0.11 \pm 0.010$ \\
\hline $9 b$ & $0.45 \pm 0.0067$ & $0.53 \pm 0.0099$ & $0.56 \pm 0.0086$ & $0.25 \pm 0.080$ \\
\hline $9 c$ & $>1$ & $>1$ & $>1$ & $>1$ \\
\hline 9d & $0.059 \pm 0.021$ & $0.11 \pm 0.024$ & $0.071 \pm 0.030$ & $0.090 \pm 0.037$ \\
\hline $9 e$ & $0.055 \pm 0.013$ & $0.080 \pm 0.0077$ & $0.059 \pm 0.0015$ & $0.057 \pm 0.014$ \\
\hline 9f & $0.062 \pm 0.015$ & $0.23 \pm 0.12$ & $0.075 \pm 0.011$ & $0.095 \pm 0.0094$ \\
\hline $9 \mathrm{~g}$ & $0.081 \pm 0.010$ & $0.27 \pm 0.12$ & $0.11 \pm 0.0011$ & $0.096 \pm 0.0056$ \\
\hline $9 h$ & $0.063 \pm 0.015$ & $0.19 \pm 0.076$ & $0.073 \pm 0.0080$ & $0.084 \pm 0.013$ \\
\hline $9 \mathrm{i}$ & $0.086 \pm 0.0065$ & $0.27 \pm 0.090$ & $0.12 \pm 0.028$ & $0.10 \pm 0.0035$ \\
\hline $9 \mathbf{j}$ & $0.058 \pm 0.012$ & $0.10 \pm 0.0024$ & $0.059 \pm 0.020$ & $0.072 \pm 0.014$ \\
\hline $9 k$ & $0.61 \pm 0.0057$ & $0.84 \pm 0.0016$ & $0.70 \pm 0.021$ & $0.74 \pm 0.031$ \\
\hline 91 & $>1$ & $>1$ & $>1$ & $>1$ \\
\hline $9 m$ & $0.17 \pm 0.087$ & $0.50 \pm 0.097$ & $0.31 \pm 0.18$ & $0.10 \pm 0.0016$ \\
\hline $9 n$ & $0.48 \pm 0.022$ & $0.61 \pm 0.0066$ & $0.58 \pm 0.013$ & $0.33 \pm 0.066$ \\
\hline 90 & $0.063 \pm 0.0021$ & $0.11 \pm 0.010$ & $0.063 \pm 0.026$ & $0.083 \pm 0.022$ \\
\hline $9 p$ & $0.062 \pm 0.0038$ & $0.091 \pm 0.0025$ & $0.080 \pm 0.020$ & $0.084 \pm 0.011$ \\
\hline $9 q$ & $0.0629 \pm 0.0061$ & $0.1027 \pm 0.0027$ & $0.0823 \pm 0.0327$ & $0.0834 \pm 0.0207$ \\
\hline $9 r$ & $0.074 \pm 0.0054$ & $0.12 \pm 0.0029$ & $0.091 \pm 0.013$ & $0.094 \pm 0.0021$ \\
\hline 9s & $0.090 \pm 0.0040$ & $0.18 \pm 0.0013$ & $0.13 \pm 0.038$ & $0.15 \pm 0.0059$ \\
\hline $9 t$ & $0.077 \pm 0.0006$ & $0.14 \pm 0.0033$ & $0.12 \pm 0.026$ & $0.095 \pm 0.0013$ \\
\hline 2 & $0.045 \pm 0.0004$ & $0.10 \pm 0.0055$ & $0.063 \pm 0.0042$ & $0.40 \pm 0.021$ \\
\hline
\end{tabular}

${ }^{a}$ A549 (lung carcinoma), MDA-MB-231 (triple-negative breast cancer), KB (originally isolated from epidermoid carcinoma of the nasopharynx), $\mathrm{KB}$ vin (vincristine-resistant $\mathrm{KB}$ subline).

${ }^{b}$ Each assay was performed in triplicate with duplicated sample, and averaged IC50 $(\mu \mathrm{M})$ values were expressed with standard deviation (SD). 